\title{
Impact of the COVID-19 pandemic in Mexican residency programs: Implications and Experiences for the Continuing Professional Development of Residents
}

Irving Armando Domínguez-Varela ( $\square$ irv_dominguez@hotmail.com )

Tecnologico de Monterrey https://orcid.org/0000-0002-2575-9334

Alejandra Celina Esparza-Sandoval

Tecnologico de Monterrey

Irving Augusto Palomares-Ramos

Tecnologico de Monterrey

María José García-Madrigal

Tecnologico de Monterrey

Jorge Eugenio Valdez-García

Tecnologico de Monterrey https://orcid.org/0000-0001-8828-720X

\section{Research Article}

Keywords: e-learning, computers, clinical skills, postgraduate, evaluation, simulation

Posted Date: October 22nd, 2021

DOI: https://doi.org/10.21203/rs.3.rs-996474/v1

License: (c) (1) This work is licensed under a Creative Commons Attribution 4.0 International License.

Read Full License 


\section{Abstract}

Introduction: The COVID-19 pandemic has forever changed the way to evaluate and teach residents, fellows, and medical students by means of new demanding learning modalities and alternatives to leverage from this new lifestyle without affecting our training as medical specialists.

As of January, 24 2021, the number of confirmed cases and deaths by COVID-19 both in Mexico and around the world have been continuously growing (1,752,347 and 149,084 vs. 99,596,451 and 2,135,068, respectively). Mexico registers the world's highest number of deaths from coronavirus among health workers, with 1,320 deaths confirmed.

Methods: Four residents of different specialties analyzed and described how the pandemic affected and benefited the ongoing residency program and exposed some recommendations for each specialty.

Results/Discussion: In Mexico, residents from all specialties are rotating in the COVID-designated area working as general practitioners exclusively for hospitalized COVID-19 patients. Our residency program has had to put a stop to routine activities such as academic sessions, specialty consultations, practices, and surgeries.

Conclusion: Nothing will replace the insights of the training from real surgical practices, but new and forgotten resources can be used to help assess surgical proficiency.

\section{Introduction}

The COVID-19 outbreak was declared as a pandemic by the World Health Organization on 11 March 2020, since then as of January 24 2021, numbers of confirmed cases and deaths around the world and Mexico have been growing (99,596,451 and 2,135,068 vs. 1,752,347 and 149,084 respectively). ${ }^{1}$ Mexico registers the world's highest number of deaths from coronavirus among health workers, with 1,320 confirmed deaths. ${ }^{2}$

In Mexico, residents from all specialties are rotating in the COVID-designated area working as general practitioners for only hospitalized COVID-19 patients. Our residency program has had to put a stop to routine activities. Four residents of the ophthalmology, internal medicine, general surgery and anesthesiology specialties shared their perspective on how the pandemic affected their training. (Table 1)

\section{Methods}

Four residents of 4 different program residencies analyzed and described how the pandemic affected and benefited the ongoing residency program. All 4 residents are from the same hospitals but different specialties. We included clinical and surgical residencies to give a greater broad.

\section{Results/discussion}




\section{Ophthalmology}

How the practice changed:

In our center, clinical and surgical ophthalmology volume abruptly decreased in April 2020 because of the COVID-19 pandemic. All the courses' workload and journal clubs migrated to an online modality and our clinical practice, along with surgical activities, decreased by $90 \%$, similar to other residency programs with a decrease in $79 \%$ of clinical visits. ${ }^{3}$ A survey on 716 ophthalmology trainees in India reported that $81 \%$ felt a negative impact to their surgical training. ${ }^{4}$

What stopped?

All elective surgeries had to be postponed, representing $95 \%$ of the surgeries performed in our residency program. Ophthalmological consultations also decreased by $90 \%$ thus reducing practice with patients for residents of our program. We performed a retrospective observational study to review the management of cataract phacoemulsification surgery (CPS) in COVID-19 times with the previous year, and we found that 240 cataract surgeries were performed in the 2020 period compared to the 643 surgeries performed in the same period of the previous year $(-62.7 \%$ in 2020 compared to $2019, p<0.0001)$. Affecting residents' learning in surgical skills.

\section{What's new?}

After finding a study reporting up to $31.6 \%$ of ocular manifestations in COVID-19 patients, we decided to start a protocol of ocular manifestations in the same hospitalized patients. ${ }^{5}$ Our program designed one weekly virtual clinical case session for residents and medical students to compensate for the lack of clinical experience at the moment. Nevertheless, 2020 has also had its bright side as the year of ophthalmology with more virtual resources being used.

\section{Internal Medicine}

How the practice changed:

Internal medicine residents were called immediately to the front line of the COVID-19 pandemic. Initially, it was unclear our role in the attention of patients; when the first cases arose in our state, we were kept out for our protection. However, when the cases exponentially increased, we became an important factor needed. Our clinical rotations continued in the hospitals that comprise our academic health center including the attention of non-COVID-19 and COVID-19 patients.

\section{What stopped?}

To ensure and enhance residents' preparation in this emerging topic, academic coursework had to focus on its pathogenesis, management innovations, and mechanical ventilation. Therefore, both clinical and academic practice had to change and became COVID-19-focused. 
It has been reported in several articles that burnout, anxiety, and stress had increased significantly in residents. ${ }^{6-8}$ The most relevant reasons are the increasing workload, the reduced sense of accomplishment, and particularly an unsafe feeling due to the risk from the exposure to transmit the virus to our families. Besides that, the pandemic has represented a big challenge to internal medicine residents because it forced us to boost our knowledge in related topics to SARS-COV-2; while, at the same time, try to continue our integral training. ${ }^{6}$

\section{General Surgery}

How the practice changed:

Our general surgery program oversees 2 hospitals that comprise our health system. One of these hospitals had to become a hospital for COVID-19 patients, so all surgical activity was stopped, and all non-COVID-19 was sent to a single hospital. Nonetheless, in the non-COVID-19 hospital was a considerable decrease on surgical activity, probably due to society's fear of contagion.

\section{What stopped?}

In the COVID-19 era, with the risk of infection and the overload of healthcare systems, we were forced to suspend elective surgeries and gradually reduce the number of admissions for surgical diseases, referring these patients to tertiary care centers. As reported, surgery residents had to join the team of residents caring for COVID-19 patients and became the main force for the placement of central venous accesses and thoracostomy. ${ }^{9}$

What's new?

Our program increased the number of online sessions of clinical cases, discussion, and research activities. The uncertainty about practical learning is vast and understandable due to the lower exposure to surgical pathologies and procedures. It is a medical specialty in which the experience and practice is essential to develop the needed skills for each procedure. ${ }^{10}$ However, we believe that with a reorganization of our learning competence systems in the following years, surgical residents will be able to recoup and achieve an adequate formation.

\section{Anesthesiology}

How the practice changed:

The pandemic has affected anesthesiology residency, in particular for not having almost any elective surgery scheduled, prioritizing obstetric, emergency, and COVID-19 related surgeries. ${ }^{11}$ This leads to very low practice and training involving general anesthesia and increasing risks of contagion. 
Until now, one of the highlights during the pandemic is the importance of airway management, and as we know, anesthesiology is a key part of the team that manages endotracheal intubation and airway procedures. $^{12,13}$

\section{What stopped?}

Considering this, guidelines have stated that only the most experienced personnel available should manage the airway of a patient with COVID-19 to minimize exposure and risks of infection ${ }^{13}$ leading anesthesiology trainees, especially first year residents, to miss the opportunity of intervention during these procedures as well as losing practice experience for endotracheal intubation.

\section{What's new?}

As well as the other specialties, we also had to join rotations in COVID-19-designated areas as general physicians. Additionally, our program supported us with training simulators and online certifications for airway management for COVID-19 patients. Worldwide adaptation to online academic sessions has also been an important part of our "new normal". ${ }^{14}$

\section{Conclusion}

The COVID-19 pandemic affected residency program differently, for better or for worse. Surgical programs decreased their surgical practice, especially for the last-year residents. For the clinical residencies they focused on analyzing pathologies with COVID-19, decreasing the academy for other pathologies. No program was prepared for this situation, these unprecedented events represented a real challenge for everyone including program managers to first-year residents. The challenge of being able to support the COVID-19 area without neglecting the main pathologies that occur by specialty and without abandoning the academic preparation of the residents, was the difficult thing the organization had to deal with.

In most specialties, the academic load increased, and all were provided with training to care for COVID-19 patients. Likewise, research and the number of publications by specialty increased considerably. Although we have already reactivated medical consultations the protection measures are maintained.

The coronavirus outbreak significantly altered and transformed medical specialty education in Mexico and accelerated the adoption of innovative virtual learning resources for trainees. These changes are here to stay and that is why new virtual learning strategies and a new way to evaluate residents' performance using virtual media are required. Residency program educators need to restructure the curricula taking in consideration that each medical specialty was affected differently. Undoubtedly, nothing will replace true surgical practices. Nonetheless, surgical skills laboratories and proctoring residents to independently performing procedures would definitely help assess surgical proficiency. 
Finally, yet importantly, the pandemic has taught us and other medical schools that we need to pay more attention to innovation and adaptation of new ways of learning. ${ }^{15}$ Using for example, simulators and technologic resources so as not to delay our training and experience.

\section{Declarations}

Acknowledgments: The authors thank Antonio García-Madrigal for his time and comments when editing this article.

Conflicts of Interest and Source Funding: The authors declare no conflict of interests nor financial disclosures.

All participants gave their consent for the publication of their information.

\section{References}

1. Worldometer. Coronavirus Cases. Worldometer. doi:10.1101/2020.01.23.20018549V2

2. México, primer lugar mundial en personal de salud fallecido por COVID-19: Amnistía Internacional. https://www.elfinanciero.com.mx/salud/mexico-primer-lugar-mundial-en-personal-de-salud-fallecidopor-covid-19-amnistia-internacional. Accessed January 24, 2021.

3. Mishra K, Boland M V., Woreta FA. Incorporating a virtual curriculum into ophthalmology education in the coronavirus disease-2019 era. Curr Opin Ophthalmol. 2020;31(5):380-385. doi:10.1097/ICU.0000000000000681

4. Mishra D, Nair A, Gandhi R, et al. The impact of COVID-19 related lockdown on ophthalmology training programs in India - Outcomes of a survey. Indian J Ophthalmol. 2020;68(6):999. doi:10.4103/ijo.IJO_1067_20

5. Wu P, Duan F, Luo C, et al. Characteristics of Ocular Findings of Patients with Coronavirus Disease 2019 (COVID-19) in Hubei Province, China. JAMA Ophthalmol. 2020;138(5):575-578. doi:10.1001/jamaophthalmol.2020.1291

6. Rakowsky S, Flashner BM, Doolin J, et al. Five questions for residency leadership in the time of COVID-19: Reflections of chief medical residents from an internal medicine program. Acad Med. 2020;95(8):1152-1154. doi:10.1097/ACM.0000000000003419

7. Milgrom Y, Richter V. Stress assessment among internal medicine residents in a level-3 hospital versus a level-2 hospital with only emergency room service for COVID-19. J Community Hosp Intern Med Perspect. 2020;10(4):301-305. doi:10.1080/20009666.2020.1782309

8. Shah K, Chaudhari G, Kamrai D, Lail A, Patel RS. How Essential Is to Focus on Physician's Health and Burnout in Coronavirus (COVID-19) Pandemic? Cureus. 2020;12(4). doi:10.7759/cureus. 7538

9. Hojaij FC, Chinelatto LA, Boog GHP, Kasmirski JA, Lopes JVZ, Sacramento FM. Surgical Practice in the Current COVID-19 Pandemic: A Rapid Systematic Review. Clinics (Sao Paulo). 2020;75:e1923. doi:10.6061/clinics/2020/e1923 
10. Collins C, Mahuron K, Bongiovanni T, Lancaster E, Sosa JA, Wick E. Stress and the Surgical Resident in the COVID-19 Pandemic. J Surg Educ. July 2020. doi:10.1016/j.jsurg.2020.07.031

11. Sneyd JR, Mathoulin SE, O'Sullivan EP, et al. Impact of the COVID-19 pandemic on anaesthesia trainees and their training. Br J Anaesth. 2020;125(4):450-455. doi:10.1016/j.bja.2020.07.011

12. Weissman DN, De Perio MA, Radonovich LJ. COVID-19 and Risks Posed to Personnel during Endotracheal Intubation. JAMA - J Am Med Assoc. 2020;323(20):2027-2028. doi:10.1001/jama.2020.6627

13. Cook TM, El-Boghdadly K, McGuire B, McNarry AF, Patel A, Higgs A. Consensus guidelines for managing the airway in patients with COVID-19: Guidelines from the Difficult Airway Society, the Association of Anaesthetists the Intensive Care Society, the Faculty of Intensive Care Medicine and the Royal College of Anaesthetists. Anaesthesia. 2020;75(6):785-799. doi:10.1111/anae.15054

14. Ferrel MN, Ryan JJ. The Impact of COVID-19 on Medical Education. Cureus. 2020;12(3). doi:10.7759/cureus.7492

15. Ahmed H, Allaf M, Elghazaly H. COVID-19 and medical education. Lancet Infect Dis. 2020;20(7):777778. doi:10.1016/S1473-3099(20)30226-7

\section{Table 1}

\section{TABLE 1.}

Residency program and its duration

\begin{tabular}{|ll|}
\hline Residency program & Years of the program \\
\hline - Ophthalmology & 4 years \\
\hline - Internal medicine & 4 years \\
\hline - General Surgery & 5 years \\
\hline - Anesthesiology & 4 years \\
\hline
\end{tabular}

